\title{
Research Paper:
}

\section{Prevention of Lung Complications following Paraquat Poisoning by Si- \\ lymarin, N-acetyl Cysteine and Hydrocortisone: An Experimental Study}

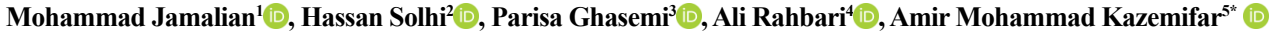 \\ 1. Clinical Toxicologist \& Assistant Professor, Department of Forensic Medicine, School of Medicine, Arak University of Medical Sciences, Arak, Iran \\ 2. Clinical Toxicologist \& Professor, Department of Forensic Medicine, School of Medicine, Arak University of Medical Sciences, Arak, Iran. \\ 3. Medical Practitioner, Department of Pathology, School of Medicine, Arak University of Medical Sciences, Arak, Iran. \\ 4. Pathologist \& Assistant Professor, Department of Forensic Medicine, School of Medicine, Arak University of Medical Sciences, Arak, Iran \\ 5. Clinical Toxicologist \& Associate Professor, Metabolic Diseases Research Center, Qazvin University of Medical Sciences, Qazvin, Iran.
}

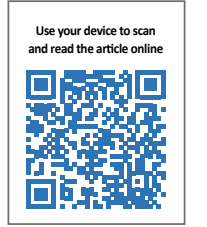

How to cite this paper Jamalian M, Solhi H, Ghasemi P, Rahbari A, Kazemifar AM. Prevention of Lung Complications following Paraquat Poisoning by Silymarin, N-acetyl Cysteine and Hydrocortisone: An Experimental Study. Iranian Journal of Toxicology. 2020; 14(4):193-200. http://dx.doi.org/10.32598/ijt.14.4.710.1

i) $h$ ttp://dx.doi.org/10.32598/ijt.14.4.710.1

\section{(i) (3)}

Article info:

Received: 05 Jun 2020

Accepted: 16 Aug 2020

Online Published: 01 Oct 2020

* Corresponding author:

Amir Mohammad Kazemifar, PhD.

Address: Metabolic Diseases Research Center, Qazvin University of Medical Sciences, Qazvin, Iran.

E-mail:am.kazemifar@gmail.com

\section{ABSTRACT}

Background: Paraquat poisoning results in multi-organ failure, primarily pulmonary fibrosis, acute renal failure, and hepatic impairment. The present study was designed to evaluate three treatment regimens, such as N-Acetyl cysteine (NAC), silymarin and hydrocortisone in the prevention of lung fibrosis after ingestion of toxic doses of paraquat in rats.

Methods: Male Sprague-Dawley rats $(\mathrm{N}=20)$ were randomly divided into four groups of five each. The drugs and paraquat were given to the rats orally. All rat groups received one oral dose of paraquat $(10 \mathrm{mg} / \mathrm{kg})$ once daily for 1 week. The first group received a daily oral dose of silymarin $(600 \mathrm{mg} / \mathrm{kg})$ for 2 weeks. The second group received a daily oral dose of NAC $(500 \mathrm{mg} / \mathrm{kg}$ ) for 2 weeks. The third group was given daily oral doses of NAC $(500 \mathrm{mg} / \mathrm{kg}$ ) and hydrocortisone $(50 \mathrm{mg} / \mathrm{kg})$ for 2 weeks. The fourth group (controls) received no drugs other than paraquat. The experiment continued for 4 weeks. After the experiment, autopsy was performed on all rats and the lungs were examined histopathologically.

Results: The results of histopathology examinations for peribronchial inflammation in the groups were shown that NAC plus hydrocortisone and silymarin had notable effects in the prevention of lung inflammation. Septal widening in the lungs was also observed in group three less than that in the other groups.

Conclusion: Based on the results, silymarin, NAC and hydrocortisone may be used as a palliative treatment in paraquat poisoning specifically aimed at preventing the acute and chronic lung injuries as the worst complication of the poisoning.

Keywords: Paraquat, Lung fibrosis, N-acetyl cysteine, Hydrocortisone, Silymarin

\section{Introduction}

araquat (1, 1'-dimethyl-4 4'-bipyridinium dichloride) is one of the most popular bipyridylium herbicides with unusual toxicological properties. This herbicide is one of the most common and active chemicals used in the Iranian agriculture [1] Paraquat poisoning results in such multi-organ failures as pulmonary fibrosis, acute renal failure, and hepatic impairment, and is associated with high mortality [2]. Toxicological properties of paraquat are attributed to its ability to produce Reactive Oxygen Species (ROS), such as superoxide anion, which may directly or indirectly 
cause cell death [3]. Lungs are the main target organ in paraquat poisoning. The main reason for death following paraquat poisoning is the resultant damage and fibrosis of the lung tissue within a few weeks post exposure [4]. It has also been reported that paraquat specifically affects the lungs in animals. In rodent models, prolonged paraquat exposure leads to cumulative and persistent damage in the brain, lungs and liver tissues [5]. Paraquat toxicity is diagnosed by the progressive interstitial fibrosis and edema of the lungs [4].

Paraquat exerts its effect as an electron donor and transforms oxygen molecules to free radicals, which are responsible for the peroxidation of lipids in cellular membranes [1]. The deleterious effects of paraquat are mainly attributed to the extreme redox activity, which in turn induces extensive damages to various organs and tissues [5]. The mechanism of cellular damage induced by paraquat is believed to be cytochrome $\mathrm{P} 450$ reductase dependent, which subsequently causes ROS formation and membrane lipid peroxidation in the pulmonary cells [4]. Oxidative stress develops when the generation of ROS in a system exceeds its ability to neutralize and eliminate the free radicals. If not regulated properly, the excess ROS can impair the cellular lipids, proteins, and/ or DNA, inhibiting their normal functions [5]. The secondary effects of oxidative stress include lipid peroxidation, mitochondrial toxicity, oxidation of Nicotinamide Adenine Dinucleotide Phosphate (NADP), apoptosis and activation of nuclear factor kappa $B$ [6]. These effects induce the formation of inflammatory enzymes, cytokines, and chemokines. Moreover, the inflammatory response is the initial and main mechanism of tissue injury secondary to paraquat poisoning [7].

No specific antidote or conclusively effective treatment has been suggested for paraquat poisoning to date [8]. In the current practice, the therapeutic regimen mainly consists of immunosuppressive agents, cyclophosphamide, and methyl prednisolone. The use of cyclophosphamide and methyl prednisolone pulse therapy is substantiated by the clinical experience of treating patients. Patients frequently present with severe lung injury, similar to those observed in systemic lupus erythematosus [9]. Evidently, severe lung injury is the primary cause of mortality in patients with paraquat poisoning [9]. The main target organ for paraquat toxicity is the lungs, involving the development of ROS, nitrogen species, inflammation, disseminated intravascular coagulation, and activation of transcriptional regulatory mechanisms. Therefore, it may be hypothesized that an antidote against paraquat poisoning should counteract all of the pathologic effects [10].
Antioxidants prevent oxidative damage caused by ROS in biological structures, and the interactions between antioxidants and toxins can reduce the toxicity [4]. Silymarin is known to exert potent antioxidative and anti-inflammatory effects on various pathophysiological processes [11].

In the present study, we have investigated the effects of three treatment regimens, i.e. N-acetyl Cysteine (NAC), silymarin, and hydrocortisone for the prevention of lung fibrosis after ingestion of toxic doses of paraquat in rats.

\section{Materials \& Methods}

Animals: Male Sprague-Dawley rats, weighing $250 \pm 10$ gr. were obtained from Razi Research Institute in Karaj, Iran. The animals were kept under standard conditions of $22 \pm 2^{\circ} \mathrm{C}, 45 \pm 5 \%$ humidity and $12 \mathrm{hr}$ light/ dark cycle. They were provided with standard laboratory diet and water ad libitum, and were left to acclimatize for 2 week before the experiments began.

Animal Grouping: Rats were randomly divided into four groups of five animals each. The drugs and paraquat were given orally through an orogastric cannula. All groups received the oral dose of paraquat $(10 \mathrm{mg} / \mathrm{kg})$ once daily for 1 week. The first group received a daily oral dose of silymarin $(600 \mathrm{mg} / \mathrm{kg})$ for 2 weeks. The second group received a daily oral dose of NAC $(500 \mathrm{mg} /$ $\mathrm{kg}$ ) for 2 weeks. The third group was given a daily oral dose of combined NAC $(500 \mathrm{mg} / \mathrm{kg})$ and hydrocortisone $(50 \mathrm{mg} / \mathrm{kg})$ for 2 weeks. The fourth group, which served as the controls, received no drugs other than paraquat.

Experimental Design: All rats were kept in animal cages (4 per cage) during the study, which was four weeks. At the completion of the experiments, all of the surviving rats were anesthetized with intramuscular injection of ketamine $(80 \mathrm{mg} / \mathrm{kg})$ and xylazine $(15 \mathrm{mg} / \mathrm{kg})$, then the lungs were removed and examined histopathologically.

Ethical Approval: The experimental protocol was approved by the Animal Care Committee at Arak's University of Medical Sciences, Arak, Iran. Also, the experimental procedures were carried out in accordance with international guidelines for the care and use of laboratory animals [12].

Histopathologic Examinations: For histopathology analysis, we prepared sections of the middle of the left lung and the lower lobe of the right lung for light microscopy. The specimen processing consisted of fixation 
in $4 \%$ buffered neutral formalin solution for $24^{\mathrm{hr}}$, embedding in paraffin wax, slicing sections at 5 micrometers thickness, and staining with Hematoxylin-Eosin (H\&E). The slides were independently examined for peribronchial inflammation and septal widening; while the examiner was unaware of the slide groups to which the specimens belonged. The extent of the tissue alterations was expressed as the mean of 10 high power fields (HPFs) under microsope, chosen at random and classified on a scale of zero to three as follows: $0=$ no change; $1=$ changes more than $15 \%$ but less than $39 \%$ of the cells; $2=$ changes more than $40 \%$ but less than $59 \%$ of the cells; $3=$ changes in more than $60 \%$ of the cells.

Statistical Analyses: We used SPSS software, v. 20 to perform all of the statistical analyses. One-way Analysis of Variance (ANOVA) and chi-square were used to test significant differences among the groups. The results were expressed as Mean \pm SD. A probability level of $<0.05$ was considered as being statistically significant.

\section{Results}

In the present study, 20 Sprague-Dawley rats were treated with paraquat and three other chemical compounds. The animals in the four study groups had no differences with respect to their body weight and living condition during the experiments. Two animals in the control group died during the study, possibly due to the paraquat poisoning. The lungs from these animals were also examined as part of the experimental samples.

Table 1. The extent of peribronchial inflammation in the rat lungs

\begin{tabular}{ccccc}
\hline \multirow{2}{*}{ Groups } & \multicolumn{4}{c}{ Peribronchial Inflammation Grade } \\
\cline { 2 - 5 } & A & B & C & D \\
\hline Group 1 & 0 & 0 & 4 & 1 \\
\hline Group 2 & 1 & 0 & 1 & 3 \\
\hline Group 3 & 1 & 2 & 2 & 5 \\
\hline Group 4* & 0 & 0 & 0 & 5 \\
\hline
\end{tabular}

A: No change; Cellular changes: $B=15-39 \% ; C=40-59 \%$; $>$ > 60\% . *Control group

Table 2. Significant statistical differences among the rats for the degree of the lung's peribronchial inflammation

\begin{tabular}{ccccc}
\hline Groups & \multicolumn{4}{c}{ P for Statistical Significance } \\
\hline Group 1 & - & $* 0.047$ & 0.097 & $* 0.031$ \\
Group 2 & $* 0.047$ & - & $* 0.039$ & 0.115 \\
Group 3 & $* 0.097$ & $* 0.039$ & - & $* 0.027$ \\
Group 4 & $* 0.031$ & 0.115 & $* 0.027$ & - \\
\hline
\end{tabular}

* Significant statistical difference

Table 3. The extent of septal widening observed in the rat lungs

\begin{tabular}{ccccc}
\hline \multirow{2}{*}{ Groups } & \multicolumn{5}{c}{ Septal Widening Grade } \\
\cline { 2 - 5 } & A & B & C & D \\
\hline Group 1 & 0 & 2 & 0 & 3 \\
\hline Group 2 & 0 & 1 & 1 & 0 \\
\hline Group 3 & 0 & 4 & 1 & 5 \\
\hline *Group 4 & 0 & 0 & 0 & 3 \\
\hline
\end{tabular}

A: No change; Cellular changes: $B=15-39 \% ; C=40-59 \%$; $>60 \%$. ${ }^{*}$ Control group 
Table 4. Significant statistical differences among the rats for the extent of the lung's septal widening

\begin{tabular}{ccccc}
\hline Groups & \multicolumn{4}{c}{ P for Statistical Significance of the Differences } \\
\hline Group 1 & - & 0.840 & $* 0.044$ & 0.243 \\
Group 2 & 0.840 & - & $0.041^{*}$ & 0.121 \\
Group 3 & $* 0.044$ & $* 0.041$ & - & $* 0.013$ \\
Group 4 & 0.243 & 0.121 & $0.013 *$ & - \\
\hline
\end{tabular}

*Significant statistical difference

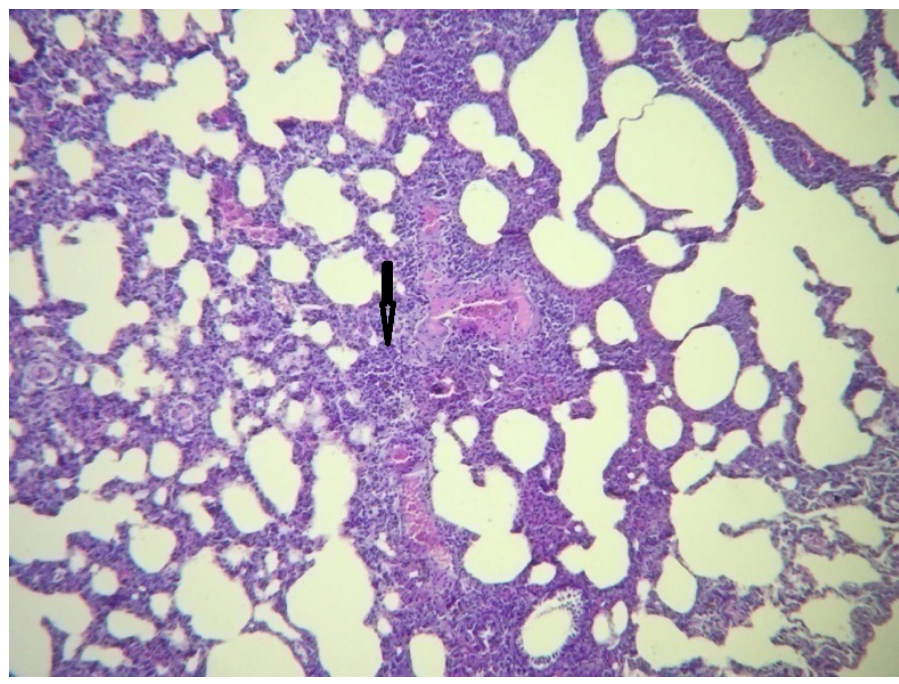

Figure 1. Severe peribronchial inflammation due to paraquat poisoning (Controls, Group 4)

The results of the histopathology examinations for peribronchial inflammation in the groups are presented in Table 1. The control group exhibited severe peribronchial inflammation. The treatments reduced the degree of the inflammation especially in groups 2 and 3 (Figures 1, $2 \& 3$ ). The significant differences among the groups for the extent of the peribronchial inflammation are shown in Table 2.

The lung's specimens were also examined for septal widening histopathologically. The results are shown in Table 3. The control group had severe septal widening

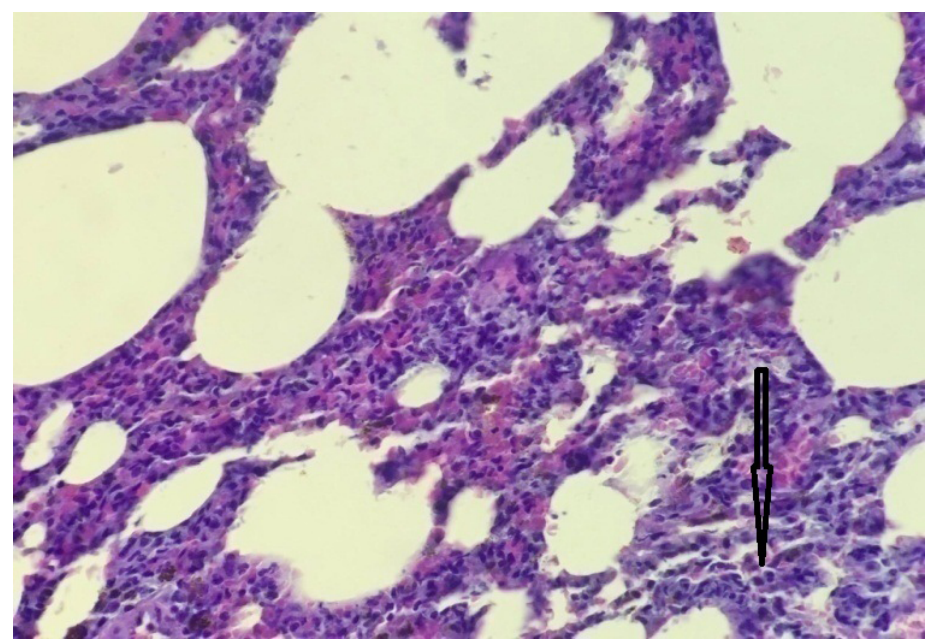

Figure 2. Moderate peribronchial inflammation due to paraquat poisoning and treatment with NAC $(500 \mathrm{mg} / \mathrm{kg})$ for 2 weeks (Group 2$)$ 


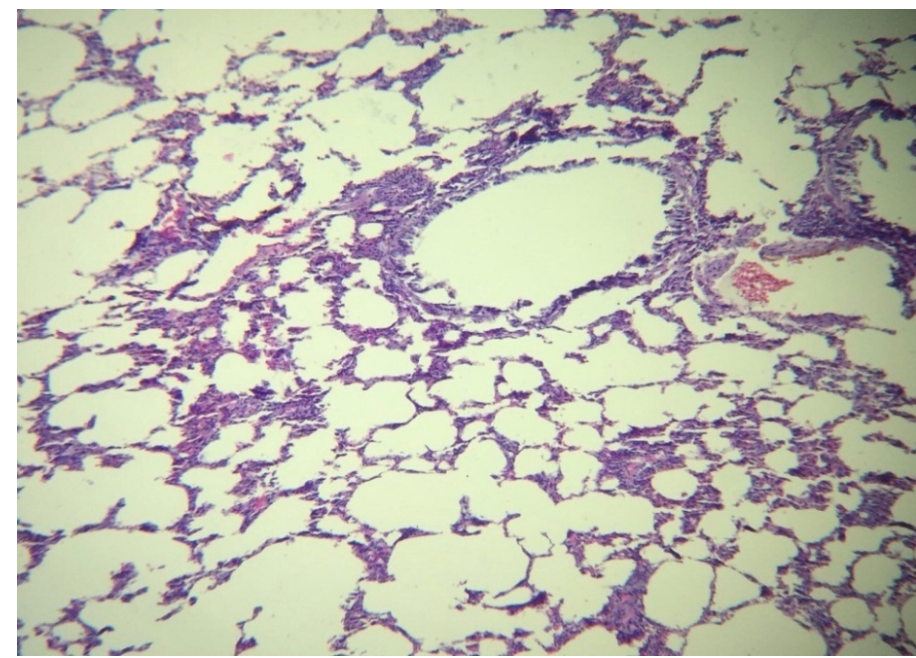

Figure 3. Mild peribronchial inflammation due to paraquat poisoning and treatment with a daily oral dose of combined NAC (500 mg/ $\mathrm{kg}$ ) and hydrocortisone (50 mg/ $\mathrm{kg}$ ) for 2 weeks (Group 3)

in the lungs. The treatment reduced the severity of the inflammation especially in group 3 . The statistical significant differences among the groups for the extent of septal widening are demonstrated in Table 4 .

\section{Discussion}

This study was designed to evaluate three treatment modalities versus lung injury in rats poisoned with paraquat. The lung tissue injuries were assessed in terms of inflammation and septal widening in each slide. The results confirmed that the proposed drug regimens were effective in preventing lung tissue injuries secondary to paraquat toxicity. The preventive effect was more prominent in the treatment where hydrocortisone and NAC had been combined. The treatment with silymarin alone was also effective moderately.

The role of oxidative stress in paraquat poisoning has been reported previously. Sharifi Nasab, et al. [1] have confirmed that the antioxidant property of vitamin $\mathrm{C}$ can alleviate the oxidative stress of paraquat on the liver. Balazadeh et al. [4] have shown the protective effects of the antioxidant, Coenzyme Q10, against lung tissue inflammation and alveoli destruction. Ahmed and colleagues have presented promising results for the antioxidant effects of Propolis in the prevention of the oxidative stress due to paraquat poisoning [5]. Charao et al. have shown significant antioxidant property of melatonin in paraquat poisoning [13]. The oxidative injuries of paraquat have also been demonstrated due to its chronic exposure [14-18].

Further, prevention of the inflammatory response and immune suppression has potential benefits in paraquat poisoning [2]. Hsin-Liang and coworkers confirmed that the presence of systemic inflammatory response was significantly associated with early mortality in paraquat poisoning [6]. Ning et al. have suggested that C-Reactive Protein (CRP) level, as a determinant of the extent of inflammation, is prognostic in paraquat poisoning [7]. Jie Qian et al. have demonstrated that an immune modulator (FTY720,) can prevent lung injuries in cases of paraquat poisoning [14].

To date, there are no effective and specific antidotes for acute poisoning with paraquat. In this context, experimentation with agents that may have beneficial effects seems prudent. Antioxidant and anti-inflammatory effects of silymarin has been studied previously by researchers in different cases of poisonings [19-21]. Silymarin and NAC have been studied in paraquat poisoning in few previous studies. Zhenning et al. have explored the protective effect of silymarin in paraquat-induced macrophage injuries with promising results [11]. TingYuan et al. have suggested that NAC treatment significantly increases the likelihood of survival in paraquatintoxicated rats [22].

Results of the present study confirmed that the combination of hydrocortisone and NAC had noticeable effect in the prevention of lung injury due to paraquat poisoning. This finding is consistent with those of previous studies $[6,7,22]$. The antioxidant and anti-inflammatory effects of these two drugs seem to improve when combined, as compared to their use individually. 
Silymarin as an herbal drug also has both antioxidant and anti-inflammatory properties. We used silymarin in the present study owing to the promising results reported in various other cases of poisoning [19-21]. Also, the outcome of the present study with silymarin alone is supportive evidence. Silymarin has been proposed for the treatment of paraquat poisoning previously by Zhenning et al. [11]. These authors have suggested that silymarin attenuates paraquat-induced cytotoxicity by inhibiting the oxidative stress, inflammation, and activation of certain cytokines [11].

\section{Conclusions}

Based on the findings of this study, silymarin, NAC and hydrocortisone have antioxidant and anti-inflammatory properties, thus they can be used as valuable treatment in patients with paraquat poisoning. Specifically, these agents prevent the acute and chronic lung tissue injuries, which are the worst and potentially lethal consequences of paraquat poisoning.

\section{Ethical Considerations}

\section{Compliance with ethical guidelines}

All ethical principles were considered in this article. The participants were informed about the purpose of the research and its implementation stages; they were also assured about the confidentiality of their information; Moreover, They were allowed to leave the study whenever they wish, and if desired, the results of the research would be available to them.

\section{Funding}

This study was funded by the Office of Research Deputy at Arak University of Medical Sciences

\section{Author's contributions}

Writing - original draft and planning: Hassan Solhi, Amir Mohammad Kazemifar; Pathologyc Study: Ali Rahbari; Data analyses and paper approvement: Mohammad Jamalian.

\section{Conflict of interest}

The authors declared no conflict of interest.

\section{Acknowledgements}

The authors extend their appreciation to the Office of Research Deputy at Arak University of Medical Scienc- es for their cooperation and support during the conduction of this study.

\section{References}

[1] Sharifinasab Z, Banaee M, Mohiseni M, Noori A. [Vitamin $\mathrm{C}$ and chitosan alleviate toxic effects of paraquat on some biochemical parameters in hepatocytes of common carp (Persian)]. Iran J Toxicol. 2016; 10 (1):31-40. http://ijt.arakmu. ac.ir/article-1-430-en.html

[2] Koh KH, Tan CHH, Hii LWS, Lee J, Ngu LLS, Chi AJM, et al. Survival predictors in paraquat intoxication and role of immunosuppression. Toxicol Rep. 2014; 1:490-5. [DOI:10.1016/j. toxrep.2014.06.010] [PMID] [PMCID]

[3] Banaee M, Nemadoost Haghi B, Tahery S, Shahafve SH, Vaziryan M. Effects of sub-lethal toxicity of paraquat on blood biochemical parameters of common carp, Cyprinus carpio (Linnaeus, 1758). Iran J Toxicol. 2016; 10(6):1-5. [DOI:10.29252/arakmu.10.6.1]

[4] Balazadeh F, Shahrooz R, Shalizar Jalali A, Karimi H, Zadeh Hashem, E. Histomorphometrical and stereological study of the lungs in neonatal rats born to paraquat-poisoned pregnant rats: Protective effect of coenzyme Q10. Iran J Toxicol. 2020; 14(2):71-80. [DOI:10.32598/ijt.14.2.581]

[5] Abass AO, Kamel NN, Khalifa WH, Gouda GF, El-Manylawi MAF, Mehaisen, GMK, et al. Propolis supplementation attenuates the negative effects of oxidative stress induced by paraquat injection on productive performance and immune function in Turkey poults. Poult Sci. 2017; 96(12):4419-29. [DOI:10.3382/ps/pex248] [PMID] [PMCID]

[6] Liu HL, Chen WL, Yang MC, Lin HM, Chou CC, Chang CF, et al. Prediction of early mortality in patients with paraquat intoxication. J Acute Med. 2013; 3(1):6-10. [DOI:10.1016/j. jacme.2013.01.001]

[7] Ning Z, Bai YL, Lu H, Mo KL. Prognostic value of plasma $\mathrm{C}$-reactive protein in the evaluation of paraquat poisoning patients. Asian Pac J Trop Bio. 2015; 5(10):841-4. [DOI:10.1016/j. apjtb.2015.06.011]

[8] Sun IO, Shin SH, Yoon HJ, Lee KY. Predicting the probability of survival in acute paraquat poisoning. Kidney Research and Clinical Practice. 2016; 35(2):102-6. [DOI:10.1016/j. krcp.2016.01.003] [PMID] [PMCID]

[9] Kuo FC, Wu MR, Hsiao CY, Chen CY, Wang KT, Yeh HI, et al. Palliative care of patients with paraquat poisoning: analysis of 90 cases from 2005-2016. Int J Gerontol. 2018; 12(3):21821. [DOI:10.1016/j.ijge.2018.02.009]

[10] Dinis-Oliveira RJ, Sousa C, Remiao F, Duarte JA, Sánchez Navarro A, Bastos ML, et al. Full survival of paraquatexposed rats after treatment with sodium salicylate. Free Radic Biol Med. 2007; 42(7):1017-28. [DOI:10.1016/j.freeradbiomed.2006.12.031] [PMID]

[11] Liu Z, Sun M, Wang Y, Zhang L, Zhao H, Zhao M. Silymarin attenuated paraquat-induced cytotoxicity in macrophages by regulating Trx/TXNIP complex, inhibiting NLRP3 inflam- 
masome activation and apoptosis. Toxicol Vitro. 2018; 46:26572. [DOI:10.1016/j.tiv.2017.10.017] [PMID]

[12] National Research Council (US) Committee for the Update of the Guide for the Care and Use of Laboratory Animals. Guide for the care and use of laboratory animals. 8th ed. Washington (DC): National Academies Press (US); 2011. [DOI:10.17226/12910] [PMID]

[13] Qian J, Ye Y, Lv L, Zhu C, Ye S. FTY720 attenuates paraquat-induced lung injury in mice. Int Immunopharmacol. 2014; 21(2):426-31. [DOI:10.1016/j.intimp.2014.05.025] [PMID]

[14] Shukla S, Singh D, Kumar V, Chauhan AK, Singh S, Ahmad I, et al. NADPH oxidase mediated maneb- and paraquatinduced oxidative stress in rat polymorphs: Crosstalk with mitochondrial dysfunction. Pestic Biochem Physiol. 2015; 123:74-86. [DOI:10.1016/j.pestbp.2015.03.007] [PMID]

[15] Charão MF, Baierle M, Gauer B, Goethel G, Fracasso R, Paese K, et al. Protective effects of melatonin-loaded lipidcore nanocapsules on paraquat-induced cytotoxicity and genotoxicity in a pulmonary cell line. Mutat Res Genet Toxicol Environ Mutagen. 2015; 784-785:1-9. [DOI:10.1016/j.mrgentox.2015.04.006] [PMID]

[16] Izumi Y, Yamamoto N, Matsushima S, Yamamoto T, Takada-Takatori $Y$, Akaike A, et al. Compensatory role of the Nrf2-ARE pathway against paraquat toxicity: Relevance of 26S proteasome activity. J Pharmacol Sci. 2015; 129(3):150-9. [DOI:10.1016/j.jphs.2015.09.003] [PMID]

[17] Rudyk C, Dwyer Z, McNeill J, Salmaso N, Farmer K, Prowse N, et al. Chronic, unpredictable stress influenced the behavioral but not the neurodegenerative impact of paraquat. Neurobiol Stress. 2019; 11:100179. [DOI:10.1016/j.ynstr.2019.100179] [PMID] [PMCID]

[18] Sambon M, Napp A, Demelenne A, Vignisse J, Wins P, Fillet $\mathrm{M}$, et al. Thiamine and benfotiamine protect neuroblastoma cells against paraquat and $\beta$-amyloid toxicity by a coenzyme-independent mechanism. Heliyon. 2019; 5(5):e01710. [DOI:10.1016/j.heliyon.2019.e01710] [PMID] [PMCID]

[19] Mohaghegh F, Solhi H, Kazemifar AM. Silymarin (milk thistle) can revoke liver enzyme changes during chemotherapy of breast cancer with Taxanes. Eur J Integr Med. 2015; 7(6):650-2. [DOI:10.1016/j.eujim.2015.10.012]

[20] Solhi H, Ghahremani R, Kazemifar AM, Hoseini Yazdi, Z. Silymarin in treatment of non-alcoholic steato-hepatitis: A randomized clinical trial. Caspian J Intern Med. 2014; 5(1):912. [PMID] [PMCID]

[21] Kazemifar AM, Hajaghamohammadi AA, Samimi R, Alavi Z, Abbasi E, Nasiri Asl M. Hepatoprotective property of oral silymarin is comparable to n-acetyl cysteine in acetaminophen poisoning. Gastroenterology Res. 2012; 5(5):190-4. [DOI:10.4021/gr463e] [PMID] [PMCID]

[22] Yeh ST, Guo HR, Su YS, Lin HJ, Hou CC, Chen HM, et al. Protective effects of $\mathrm{N}$-acetylcysteine treatment post acute paraquat intoxication in rats and in human lung epithelial cells. Toxicology. 2006; 223(3):181-90. [DOI:10.1016/j. tox.2006.03.019] [PMID] 
This Page Intentionally Left Blank 